\title{
Numerical approach for high precision 3-D relativistic star models
}

\author{
Silvano Bonazzola, Eric Gourgoulhon and Jean-Alain Marck \\ Département d'Astrophysique Relativiste et de Cosmologie \\ UPR 176 du C.N.R.S., Observatoire de Paris, \\ F-92195 Meudon Cedex, France \\ e-mail: bona@mesiob.obspm.fr, Eric.Gourgoulhon@obspm.fr, Jean-Alain.Marck@obspm.fr
}

(8 June 1998)

\begin{abstract}
A multi-domain spectral method for computing very high precision 3-D stellar models is presented. The boundary of each domain is chosen in order to coincide with a physical discontinuity (e.g. the star's surface). In addition, a regularization procedure is introduced to deal with the infinite derivatives on the boundary that may appear in the density field when stiff equations of state are used. Consequently all the physical fields are smooth functions on each domain and the spectral method is absolutely free of any Gibbs phenomenon, which yields to a very high precision. The power of this method is demonstrated by direct comparison with analytical solutions such as MacLaurin spheroids and Roche ellipsoids. The relative numerical error reveals to be of the order of $10^{-10}$. This approach has been developed for the study of relativistic inspiralling binaries. It may be applied to a wider class of astrophysical problems such as the study of relativistic rotating stars too.
\end{abstract}

PACS number(s): 02.60.Cb 02.70.Hm 04.25.Dm

\section{INTRODUCTION}

One of the most promising source of gravitational waves is the coalescence of inspiralling compact binaries. The recent development of interferometric gravitational waves detectors (e.g. GEO600, LIGO, TAMA and VIRGO) gives an important motivation for studying this problem. Such a study requires a relativistic formalism to derive the equations of motion and then an accurate and tricky method to solve the resulting system of partial differential equations. We have recently [1] proposed a relativistic formalism able to tackle the problem of co-rotating as well as counter-rotating binaries system, these latter being more relevant from the astrophysical point of view. We present now a very accurate approach based on multi-domain spectral method that circumvents the Gibbs phenomenon to numerically solve this problem and which can be applied to a wide class of other astrophysical situations.

Various astrophysical applications of spectral methods have been developed in our group (for a review, see [2]), including 3-D gravitational collapse of stellar core [3], neutron star collapse into a black hole [4], tidal disruption of a star near a massive black hole [8], rapidly rotating neutron stars [9] 12], magnetized neutron stars [13,14 and their resulting gravitational radiation [15, spontaneous symmetry breaking of rapidly rotating neutron stars [16, 17], proto-neutron stars evolution [18]20].

In computational fluid dynamics, spectral methods are known for their very high accuracy [21,22]; indeed for a $\mathcal{C}^{\infty}$ function, the numerical error decreases as $\exp (-N)$ (evanescent error), where $N$ is the number of coefficients involved in the spectral expansion, or equivalently the number of grid points in the physical domain. This is much faster than the error decay of finite-difference methods, which behaves as $1 / N^{q}$, with $q$ generally not larger than 3 . For this reason, spectral methods are particularly interesting for treating 3-D problems - such as binary configurations - a situation in which the number of grid points is still severely limited by the capability of present and next generation computers.

Spectral methods lose much of their accuracy when non-smooth functions are treated because of the so-called Gibbs phenomenon. This phenomenon is well known from the most familiar spectral method, namely the theory of Fourier series: the Fourier coefficients $\left(c_{n}\right)$ of a function $f$ which is of class $\mathcal{C}^{p}$ but not $\mathcal{C}^{p+1}$ decrease as $1 / n^{p}$ only. In particular, if the function has some discontinuity, its approximation by a Fourier series does not converge towards $f$ at the discontinuity point: there remains a gap which is of the order $10 \%$.

The multi-domain spectral method described in this paper circumvents the Gibbs phenomenon. The basic idea is to divide the space into domains chosen so that the physical discontinuities are located onto the boundaries between the domains (Sect. II). The simplest example is the case of a perfect fluid star, where two domains may be distinguished: the interior and the exterior of the star. The boundary is then simply the surface of the star. The second ingredient of the technique is a mapping between the domains defined in this way and some simple mathematical domains, which are cross products of intervals: $\left[a_{1}, a_{2}\right] \times\left[b_{1}, b_{2}\right] \times\left[c_{1}, c_{2}\right]$. The spectral expansion is then performed with respect to functions of the coordinates spanning these intervals (Sect. III). The method of resolution of a basic equation, namely 
the Poisson equation, is exposed in Sect. IIIC. For stiff equations of state, the above procedure is not sufficient to ensure the smoothness of all the functions. Indeed, for a polytrope with an adiabatic index greater than 2, the density field has an infinite derivative on the surface of the star. We present in Sect. IV a method for regularizing the density and recover the spectral precision. The power of the multi-domain spectral method is illustrated in Sect. $\mathrm{V}$, where comparisons are performed between numerical solutions obtained by an implementation of the method and analytical solutions (ellipsoidal configurations of incompressible fluids). Finally Sect. VI concludes the article by discussing the great advantages of the multi-domain spectral method for dealing with relativistic binary neutron stars.

\section{THE PHYSICAL DOMAINS AND THEIR MAPPING}

\section{A. Splitting of the physical space into star-like domains}

In order to treat problems involving Poisson equations with non-compact sources - as they appear in relativistic gravitation - we take for the physical domain where the computation must be performed the whole three-dimensional space $\mathbb{R}^{3}$. In doing so, we know the physical boundary conditions we have to impose in order to solve the Poisson equations. These boundary conditions can be easily set at infinity. We divide $\mathbb{R}^{3}$ into $\mathcal{N}$ domains $\left(\mathcal{D}_{l}\right)_{0 \leq l \leq \mathcal{N}-1}$ $(\mathcal{N} \geq 2)$. In the present work, these domains are taken to be star-like (in the mathematical sense) with respect to a some origin $O$, which means that for every point $M$ in the domain $\mathcal{D}_{l}$, the segment $O M$ is entirely included in $\bigcup_{i \leq l} \mathcal{D}_{l}$ (see Fig. 11). The multi-domain spectral method we are going to describe can be extended to more general domains, at the price of a greater technical (but non conceptual) difficulty. However, for stellar configurations, the star-like hypothesis is sufficient for most applications. Let us denote by $\mathcal{S}_{l}$ the boundary surface between the domains $\mathcal{D}_{l}$ and $\mathcal{D}_{l+1} . \mathcal{D}_{0}$ is simply connected and its boundary is $\mathcal{S}_{0}$; we call it the nucleus. For $1 \leq l \leq \mathcal{N}-2$, $\mathcal{D}_{l}$ 's inner boundary is $\mathcal{S}_{l-1}$ and outer boundary $\mathcal{S}_{l}$. The last domain, $\mathcal{D}_{\mathcal{N}-1}$, has $\mathcal{S}_{\mathcal{N}-2}$ as inner boundary and extends to infinity (cf. Fig. 这).

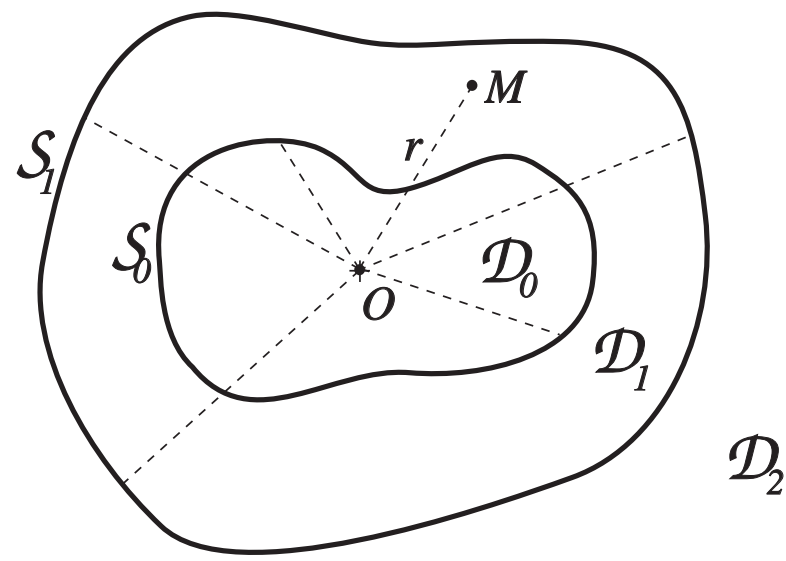

FIG. 1. Splitting of the physical three-dimensional space into domains $\mathcal{D}_{0}, \mathcal{D}_{1}, \ldots, \mathcal{D}_{\mathcal{N}-1}$ (on the figure $\mathcal{N}=3$ ), which are star-like with respect to some origin $O$. The last domain (here $\mathcal{D}_{2}$ ) extends up to infinity.

Let us choose some Cartesian frame of $\mathbb{R}^{3}$ centered at $O$ and let us call $(r, \theta, \varphi)$ the associated spherical coordinates: $r \in\left[0,+\infty\left[, \theta \in[0, \pi]\right.\right.$ and $\varphi \in\left[0,2 \pi\left[\right.\right.$. The mapping of each domain onto the cross product of intervals $\left[a_{1}, a_{2}\right] \times$ $\left[b_{1}, b_{2}\right] \times\left[c_{1}, c_{2}\right]$ will be defined with respect to the spherical coordinates $(r, \theta, \varphi)$. Since each domain $\mathcal{D}_{l}$ is star-like with respect to $O$, the equation of the boundaries $\mathcal{S}_{l}$ can be written under the form

$$
r=S_{l}(\theta, \varphi)
$$

where $S_{l}$ is a smooth function on $[0, \pi] \times[0,2 \pi[$.

\section{B. Mapping of the nucleus}

The basic idea is to introduce a mapping 


$$
\begin{aligned}
{[0,1] \times[0, \pi] \times[0,2 \pi[} & \longrightarrow \mathcal{D}_{0} \\
\left(\xi, \theta^{\prime}, \varphi^{\prime}\right) & \longmapsto(r, \theta, \varphi)
\end{aligned}
$$

so that the origin $O$ corresponds to $\xi=0$ and the boundary $\mathcal{S}_{0}$ to $\xi=$ const $=1$. Using the fact that $\mathcal{D}_{0}$ is star-like, a simple form of the mapping (2) can be chosen as

$$
\begin{aligned}
r & =R_{0}\left(\xi, \theta^{\prime}, \varphi^{\prime}\right) \\
\theta & =\theta^{\prime} \\
\varphi & =\varphi^{\prime}
\end{aligned}
$$

where $R_{0}$ is a smooth function subject to regularity properties which are discussed below. Thanks to Eqs. (4)-(河), we will make no distinction between $\theta$ and $\theta^{\prime}$, as well as between $\varphi$ and $\varphi^{\prime}$, i.e. we will abandon the primes on the angles. The fact that $\mathcal{D}_{0}$ 's boundary coincides with $\xi=1$ translates as

$$
R_{0}(1, \theta, \varphi)=S_{0}(\theta, \varphi)
$$

Beside Eq. (6), the function $R_{0}$ must satisfy some regularity properties induced by the singular behavior of spherical coordinates at $r=0, \theta=0$ and $\theta=\pi$. We define a function $f: \mathbb{R}^{3} \rightarrow \mathbb{R}$ to be regular if it can be expressed as a polynomial of the Cartesian coordinates

$$
\begin{aligned}
& x=r \sin \theta \cos \varphi \\
& y=r \sin \theta \sin \varphi \\
& z=r \cos \theta .
\end{aligned}
$$

We will assume that all the physical fields are regular functions on each domain $\mathcal{D}_{l}$ (the domains $\mathcal{D}_{l}$ are in fact chosen in this manner) with respect to the previous definition. It is easy to see that any regular function is expandable as

$$
f(r, \theta, \varphi)=\sum_{m=-M}^{M} \sum_{\ell=|m|}^{L} r^{\ell} T\left(r^{2}\right) \sin ^{|m|} \theta \quad P_{\ell-|m|}(\cos \theta) e^{i m \varphi}
$$

where $L$ and $M$ are positive integers, $L \geq M, P_{\ell-|m|}$ is some polynomial of degree $\ell-|m|$ and $T\left(r^{2}\right)$ is some even polynomial.

A simple form of the mapping (3)-(5) has been already introduced in the literature [23 25], namely $R_{0}(\xi, \theta, \varphi)=$ $S_{0}(\theta, \varphi) \xi$, where $S_{0}(\theta, \varphi)$ is the equation of the star's surface [Eq. (11)]. However for such a mapping the regularity condition (10) would be quite complicated when expressed in terms of $(\xi, \theta, \varphi)$. We choose instead the mapping defined by

$$
R_{0}(\xi, \theta, \varphi)=\alpha_{0}\left[\xi+A_{0}(\xi) F_{0}(\theta, \varphi)+B_{0}(\xi) G_{0}(\theta, \varphi)\right]
$$

where $A_{0}$ and $B_{0}$ are the following even and odd polynomials

$$
\begin{aligned}
& A_{0}(\xi)=3 \xi^{4}-2 \xi^{6} \\
& B_{0}(\xi)=\left(5 \xi^{3}-3 \xi^{5}\right) / 2
\end{aligned}
$$

and the constant $\alpha_{0}$ as well as the two functions $F_{0}(\theta, \varphi)$ and $G_{0}(\theta, \varphi)$ are such that (i) the Fourier expansion of $F_{0}(\theta, \varphi)$ (resp. $G_{0}(\theta, \varphi)$ ) with respect to $\varphi$ contains only even (resp. odd) harmonics and (ii) the equation of the surface $\mathcal{S}_{0}$ can be written

$$
\alpha_{0}\left[1+F_{0}(\theta, \varphi)+G_{0}(\theta, \varphi)\right]=S_{0}(\theta, \varphi) .
$$

The polynomials $A_{0}$ and $B_{0}$ defined by (12)-(13) are such that

$$
\begin{aligned}
& A_{0}(0)=B_{0}(0)=0 \\
& A_{0}^{\prime}(0)=B_{0}^{\prime}(0)=0 \\
& A_{0}(1)=B_{0}(1)=1 \\
& A_{0}^{\prime}(1)=B_{0}^{\prime}(1)=0 .
\end{aligned}
$$

The properties (14) and (17) ensure that Eq. (6) is satisfied, i.e. that the mapping $(11)$ is from $[0,1] \times[0, \pi] \times[0,2 \pi[$ to $\mathcal{D}_{0}$. The polynomials $A_{0}$ and $B_{0}$ are chosen in order to satisfy at the minimum level the regularity conditions 
mentioned above. In particular, near the origin $r$ behaves as $r \sim \alpha_{0} \xi$ and is independent of $(\theta, \varphi)$, which would not have been the case of the mapping $r=S_{0}(\theta, \varphi) \xi$ introduced in Refs. 23. 25.].

The Jacobian of the transformation $(r, \theta, \varphi) \mapsto(\xi, \theta, \varphi)$ is

$$
\begin{aligned}
J & :=\frac{\partial(r, \theta, \varphi)}{\partial(\xi, \theta, \varphi)}=\frac{\partial R_{0}}{\partial \xi} \\
& =\alpha_{0}\left[1+A_{0}^{\prime}(\xi) F_{0}(\theta, \varphi)+B_{0}^{\prime}(\xi) G_{0}(\theta, \varphi)\right]
\end{aligned}
$$

Since $A_{0}^{\prime}(\xi) \geq 0$ and $B_{0}^{\prime}(\xi) \geq 0$ for any $\xi \in[0,1]$, the mapping could become singular if $F_{0}(\theta, \varphi)$ or $G_{0}(\theta, \varphi)$ is negative and has an important amplitude. We cannot control the sign of the function $F_{0}(\theta, \varphi)$ because it contains only odd harmonics of $\varphi$. However, by a suitable choice of $\alpha_{0}$, one can impose

$$
G_{0}(\theta, \varphi) \geq 0,
$$

as we shall see below. We have found that this condition was sufficient to ensure that $J \neq 0$, i.e. that the mapping is regular, for all the astrophysically relevant situations we have encountered.

The equation for the surface of the domain $\mathcal{D}_{0}$ being given, under the form (1]), $r=S_{0}(\theta, \varphi)$, the procedure which leads to $\alpha_{0}, F_{0}(\theta, \varphi)$ and $G_{0}(\theta, \varphi)$, i.e. to the full determination of the function $R_{0}(\xi, \theta, \varphi)$, is the following one.

First let us choose a point $\left(\theta_{*}, \varphi_{*}\right)$ on the surface $\mathcal{S}_{0}$. Equation (14) implies the following relation

$$
\alpha_{0}\left[1+F_{0}\left(\theta_{*}, \varphi_{*}\right)+G_{0}\left(\theta_{*}, \varphi_{*}\right)\right]=S_{0}\left(\theta_{*}, \varphi_{*}\right) .
$$

Let us introduce the following auxiliaries quantities

$$
\begin{aligned}
& \mu:=\alpha_{0}\left[F_{0}\left(\theta_{*}, \varphi_{*}\right)+G_{0}\left(\theta_{*}, \varphi_{*}\right)\right] \\
& \tilde{F}_{0}(\theta, \varphi):=\alpha_{0} F_{0}(\theta, \varphi) \\
& \tilde{G}_{0}(\theta, \varphi):=\alpha_{0} G_{0}(\theta, \varphi)-\mu
\end{aligned}
$$

Equation (21) translates then in

$$
\alpha_{0}+\mu=S_{0}\left(\theta_{*}, \varphi_{*}\right)
$$

whereas Eq. (14) becomes

$$
\tilde{F}_{0}(\theta, \varphi)+\tilde{G}_{0}(\theta, \varphi)=S_{0}(\theta, \varphi)-S_{0}\left(\theta_{*}, \varphi_{*}\right) .
$$

Having expanded $f(\theta, \varphi):=S_{0}(\theta, \varphi)-S_{0}\left(\theta_{*}, \varphi_{*}\right)$ into Fourier series with respect to $\varphi$, one deduces the functions $\tilde{F}_{0}(\theta, \varphi)$ (resp. $\tilde{G}_{0}(\theta, \varphi)$ ) by taking only the odd (resp. even) harmonics of this Fourier expansion. $\mu$ is then computed as

$$
\mu=-\min \left\{\tilde{G}_{0}(\theta, \varphi),(\theta, \varphi) \in[0, \pi] \times[0,2 \pi[\} .\right.
$$

In doing so, condition (20) will automatically be fulfilled. The value of the coefficient $\alpha_{0}$ is deduced from the above value of $\mu$ via Eq. (25). Finally the functions $F_{0}(\theta, \varphi)$ and $G_{0}(\theta, \varphi)$ are computed from Eqs. (23) and (24).

\section{Mapping of the intermediate domains}

For $1 \leq l \leq \mathcal{N}-2$, we introduce the mapping

$$
\begin{aligned}
{[-1,1] \times[0, \pi] \times[0,2 \pi[} & \longrightarrow \mathcal{D}_{l} \\
\left(\xi, \theta^{\prime}, \varphi^{\prime}\right) & \longmapsto(r, \theta, \varphi)
\end{aligned}
$$

under the form

$$
\begin{aligned}
r & =R_{l}\left(\xi, \theta^{\prime}, \varphi^{\prime}\right) \\
\theta & =\theta^{\prime} \\
\varphi & =\varphi^{\prime},
\end{aligned}
$$


where $R_{l}$ is a smooth function which satisfies

$$
\begin{aligned}
& R_{l}(-1, \theta, \varphi)=S_{l-1}(\theta, \varphi) \\
& R_{l}(+1, \theta, \varphi)=S_{l}(\theta, \varphi),
\end{aligned}
$$

which means that the inner (resp. outer) boundary of $\mathcal{D}_{l}$ is defined by $\xi=-1$ (resp. $\left.\xi=+1\right)$.

We choose $R_{l}(\xi, \theta, \varphi)$ as

$$
\begin{aligned}
R_{l}(\xi, \theta, \varphi)= & \alpha_{l}\left[\xi+A_{l}(\xi) F_{l}(\theta, \varphi)+B_{l}(\xi) G_{l}(\theta, \varphi)\right] \\
& +\beta_{l},
\end{aligned}
$$

where $A_{l}$ and $B_{l}$ are the following polynomials

$$
\begin{aligned}
& A_{l}(\xi)=\left(\xi^{3}-3 \xi+2\right) / 4 \\
& B_{l}(\xi)=\left(-\xi^{3}+3 \xi+2\right) / 4,
\end{aligned}
$$

and the constants $\alpha_{l}$ and $\beta_{l}$ and the two functions $F_{l}(\theta, \varphi)$ and $G_{l}(\theta, \varphi)$ are defined from the equations of the surfaces $\mathcal{S}_{l-1}$ and $\mathcal{S}_{l}$ by

$$
\begin{aligned}
& \alpha_{l}\left[-1+F_{l}(\theta, \varphi)\right]+\beta_{l}=S_{l-1}(\theta, \varphi) \\
& \alpha_{l}\left[+1+G_{l}(\theta, \varphi)\right]+\beta_{l}=S_{l}(\theta, \varphi) \\
& F_{l}(\theta, \varphi) \leq 0 \\
& G_{l}(\theta, \varphi) \geq 0
\end{aligned}
$$

Note that the polynomials $A_{l}$ and $B_{l}$ defined by (35)-(36) are such that

$$
\begin{aligned}
& A_{l}(-1)=1 \quad \text { and } \quad B_{l}(-1)=0 \\
& A_{l}(+1)=0 \quad \text { and } \quad B_{l}(+1)=1 \\
& A_{l}^{\prime}(-1)=B_{l}^{\prime}(-1)=A_{l}^{\prime}(+1)=B_{l}^{\prime}(+1)=0 .
\end{aligned}
$$

The properties (37) and (41) [resp. (38) and (42)] ensure that Eq. (32) [resp. Eq. (33)] is satisfied, i.e. that the mapping (34) is from $[-1,1] \times[0, \pi] \times\left[0,2 \pi\left[\right.\right.$ to $\mathcal{D}_{l}$. The conditions (39) and (40) ensure that this mapping is not singular, by the same argument as that presented for $R_{0}$ in Sect. II B, the sign of $F_{l}(\theta, \varphi)$ being opposite to that of $G_{l}(\theta, \varphi)$ because $A_{l}$ is a decreasing function of $\xi$, whereas $B_{l}$ is an increasing function of $\xi$.

The equation for the inner and outer boundaries of the domain $\mathcal{D}_{l}$ being given, under the form (1): $r=S_{l-1}(\theta, \varphi)$ (inner boundary) and $r=S_{l}(\theta, \varphi)$ (outer boundary), the procedure which leads to $\alpha_{l}, \beta_{l}, F_{l}(\theta, \varphi)$ and $G_{l}(\theta, \varphi)$, i.e. to the full determination of the function $R_{l}(\xi, \theta, \varphi)$, is the following one.

First let us choose a point $\left(\theta_{*}, \varphi_{*}\right)$ on the surface $\mathcal{S}_{l-1}$ along with the corresponding point $\left(\theta_{*}, \varphi_{*}\right)$ on the surface $\mathcal{S}_{l}$. Equations (37) and (38) imply the following relations

$$
\begin{aligned}
& \alpha_{l}\left[-1+F_{l}\left(\theta_{*}, \varphi_{*}\right)\right]+\beta_{l}=S_{l-1}\left(\theta_{*}, \varphi_{*}\right) \\
& \alpha_{l}\left[1+G_{l}\left(\theta_{*}, \varphi_{*}\right)\right]+\beta_{l}=S_{l}\left(\theta_{*}, \varphi_{*}\right) .
\end{aligned}
$$

Let us introduce the following auxiliaries quantities

$$
\begin{aligned}
& \lambda:=\alpha_{l} F_{l}\left(\theta_{*}, \varphi_{*}\right) \\
& \mu:=\alpha_{l} G_{l}\left(\theta_{*}, \varphi_{*}\right) \\
& \tilde{F}_{l}(\theta, \varphi):=\alpha_{l} F_{l}(\theta, \varphi)-\lambda \\
& \tilde{G}_{l}(\theta, \varphi):=\alpha_{l} G_{l}(\theta, \varphi)-\mu .
\end{aligned}
$$

Equations (44) and (45) then translate in

$$
\begin{aligned}
& -\alpha_{l}+\lambda+\beta_{l}=S_{l-1}\left(\theta_{*}, \varphi_{*}\right) \\
& \alpha_{l}+\mu+\beta_{l}=S_{l}\left(\theta_{*}, \varphi_{*}\right),
\end{aligned}
$$

whereas Eqs. (37) and (38) become 


$$
\begin{aligned}
& \tilde{F}_{l}(\theta, \varphi)=S_{l-1}(\theta, \varphi)-S_{l-1}\left(\theta_{*}, \varphi_{*}\right) \\
& \tilde{G}_{l}(\theta, \varphi)=S_{l}(\theta, \varphi)-S_{l}\left(\theta_{*}, \varphi_{*}\right) .
\end{aligned}
$$

From the values of $\tilde{F}_{l}(\theta, \varphi)$ and $\tilde{G}_{l}(\theta, \varphi)$ obtained above, $\lambda$ and $\mu$ are computed as

$$
\begin{aligned}
& \lambda=-\max \left\{\tilde{F}_{l}(\theta, \varphi),(\theta, \varphi) \in[0, \pi] \times[0,2 \pi[\}\right. \\
& \mu=-\min \left\{\tilde{G}_{l}(\theta, \varphi),(\theta, \varphi) \in[0, \pi] \times[0,2 \pi[\} .\right.
\end{aligned}
$$

In doing so, the conditions (39) and (40) will automatically be fulfilled. The value of the constants $\alpha_{l}$ and $\beta_{l}$ are deduced from the above values of $\lambda$ and $\mu$ via Eqs. (50) and (51). Finally the functions $F_{l}(\theta, \varphi)$ and $G_{l}(\theta, \varphi)$ are computed from Eqs. (48) and (49).

\section{Compactification of the infinite domain}

In the case where the external domain $\mathcal{D}_{\text {ext }}:=\mathcal{D}_{\mathcal{N}-1}$ extends to infinity we introduce the mapping

$$
\begin{aligned}
{[-1,1] \times[0, \pi] \times[0,2 \pi[} & \longrightarrow \mathcal{D}_{\text {ext }} \\
\left(\xi, \theta^{\prime}, \varphi^{\prime}\right) & \longmapsto(r, \theta, \varphi)
\end{aligned}
$$

under the form

$$
\begin{aligned}
u & :=1 / r=U\left(\xi, \theta^{\prime}, \varphi^{\prime}\right) \\
\theta & =\theta^{\prime} \\
\varphi & =\varphi^{\prime}
\end{aligned}
$$

where $U$ is a smooth function which satisfies

$$
\begin{aligned}
& U(-1, \theta, \varphi)=S_{\text {ext }}(\theta, \varphi)^{-1} \\
& U(+1, \theta, \varphi)=0
\end{aligned}
$$

where $S_{\text {ext }}(\theta, \varphi):=S_{\mathcal{N}-2}(\theta, \varphi)$. The above two equations show that the inner boundary of $\mathcal{D}_{\text {ext }}$ is defined by $\xi=-1$, whereas $\xi=+1$ corresponds to the infinity. We have already introduced such a compactification of the infinite domain in Ref. [9], in the case of a spherical inner boundary.

We choose the function $U(\xi, \theta, \varphi)$ as

$$
U(\xi, \theta, \varphi)=\alpha_{\text {ext }}\left[\xi+A_{\text {ext }}(\xi) F_{\text {ext }}(\theta, \varphi)-1\right],
$$

where $A_{\text {ext }}$ is the same polynomial of $\xi$ as that defined in Eq. (35), and the constant $\alpha_{\text {ext }}$ and the function $F_{\text {ext }}(\theta, \varphi)$ are defined from the equations of the surface $\mathcal{S}_{\mathcal{N}-2}$ by

$$
\begin{aligned}
& \alpha_{\text {ext }}\left[-2+F_{\text {ext }}(\theta, \varphi)\right]=S_{\text {ext }}(\theta, \varphi)^{-1} \\
& F_{\text {ext }}(\theta, \varphi) \leq 0 .
\end{aligned}
$$

The condition (64) ensures that $\partial U / \partial \xi \neq 0$ i.e. that the mapping (62) is not singular.

The equation for the inner boundary of the domain $\mathcal{D}_{\text {ext }}$ being given, under the form (四): $r=S_{\text {ext }}(\theta, \varphi)$, the procedure which leads to $\alpha_{\text {ext }}$ and $F_{\text {ext }}(\theta, \varphi)$, i.e. to the full determination of the function $U(\xi, \theta, \varphi)$, is the following one. First let us choose a point $\left(\theta_{*}, \varphi_{*}\right)$ on the surface $\mathcal{S}_{\text {ext }}$. Equation (63) implies the following relation

$$
\alpha_{\text {ext }}\left[-2+F_{\text {ext }}\left(\theta_{*}, \varphi_{*}\right)\right]=S_{\text {ext }}\left(\theta_{*}, \varphi_{*}\right)^{-1} .
$$

By introducing the auxiliaries quantities

$$
\begin{aligned}
& \lambda:=\alpha_{\text {ext }} F_{\text {ext }}\left(\theta_{*}, \varphi_{*}\right) \\
& \tilde{F}_{\text {ext }}(\theta, \varphi):=\alpha_{\text {ext }} F_{\text {ext }}(\theta, \varphi)-\lambda,
\end{aligned}
$$

this equation translates in 


$$
-2 \alpha_{\mathrm{ext}}+\lambda=S_{\mathrm{ext}}\left(\theta_{*}, \varphi_{*}\right)^{-1}
$$

whereas Eq. (63) becomes

$$
\tilde{F}_{\text {ext }}(\theta, \varphi)=S_{\text {ext }}(\theta, \varphi)^{-1}-S_{\text {ext }}\left(\theta_{*}, \varphi_{*}\right)^{-1}
$$

From the above value of $\tilde{F}_{\text {ext }}(\theta, \varphi), \lambda$ is computed according to

$$
\lambda=-\min \left\{\tilde{F}_{\text {ext }}(\theta, \varphi),(\theta, \varphi) \in[0, \pi] \times[0,2 \pi[\} .\right.
$$

In doing so, the condition (64) will automatically be fulfilled (recall that $\alpha_{\text {ext }}<0$ ). The value of $\alpha_{\text {ext }}$ is deduced from the above value of $\lambda$ via Eq. (68). Finally the function $F_{\text {ext }}(\theta, \varphi)$ is computed from Eq. (67).

\section{MULTI-DOMAIN SPECTRAL METHOD}

\section{A. Spectral expansion of a physical field}

The spirit of the multi-domain spectral method is to perform spectral expansions on each domain $\mathcal{D}_{l}$, and with respect to the coordinates $(\xi, \theta, \varphi)$ instead of the physical coordinates $(r, \theta, \varphi)$. We shall take as basis functions separable functions of $(\xi, \theta, \varphi)$, i.e. functions that can be put under the form $X(\xi) \Theta(\theta) \Phi(\varphi)$. The variable $\varphi$ being periodic, it is natural to use Fourier series in $\varphi$, i.e. to choose

$$
\Phi_{k}(\varphi)=e^{i k \varphi} \quad-N_{\varphi} / 2 \leq k \leq N_{\varphi} / 2
$$

where $N_{\varphi}$ is an even integer that we will call the number of degrees of freedom in $\varphi$. The associated collocation points ("grid points") are

$$
\varphi_{k}=2 \pi k / N_{\varphi} \quad 0 \leq k \leq N_{\varphi}-1
$$

As concerns $\Theta(\theta)$ one must use functions that are compatible with the expansion (10) of any regular scalar field $f$. We shall not use $\sin ^{|m|} \theta P_{\ell-|m|}(\cos \theta)$, as suggested by Eq. (10), but a wider set, namely the functions

$$
\begin{array}{lll}
\Theta_{k j}(\theta)=\cos (j \theta) & 0 \leq j \leq N_{\theta}-1 & \text { for } m \text { even } \\
\Theta_{k j}(\theta)=\sin (j \theta) & 1 \leq j \leq N_{\theta}-2
\end{array} \text { for } m \text { odd },
$$

where $N_{\theta}$ is an integer that we will call the number of degrees of freedom in $\theta$ and $m$ is the degree of the harmonic in the Fourier series with respect to $\varphi: m=k$ in the present case. The advantages of this choice are to allow the use of Fast-Fourier-Transform algorithms for computing the coefficients, as well as very simple matrices for the usual differential operators [26]. The associated collocation points are

$$
\theta_{j}=\pi j /\left(N_{\theta}-1\right) \quad 0 \leq j \leq N_{\theta}-1
$$

As concerns the variable $\xi$, we also choose a set wider than merely $\xi^{\ell}$, namely:

$$
\begin{aligned}
& X_{k j i}(\xi)=T_{2 i}(\xi) \quad 0 \leq i \leq N_{r}-1 \quad \text { for } j \text { even } \\
& X_{k j i}(\xi)=T_{2 i+1}(\xi) \quad 0 \leq i \leq N_{r}-2 \text { for } j \text { odd }
\end{aligned}
$$

where $N_{r}$ is an integer that we will call the number of degrees of freedom in $r$ and $T_{n}$ denotes the $n^{\text {th }}$ degree Chebyshev polynomial. The associated collocation points are

$$
\xi_{i}=\sin \left(\frac{\pi}{2} \frac{i}{N_{r}-1}\right) \quad 0 \leq i \leq N_{r}-1 .
$$

The above choice concerns the nucleus $\mathcal{D}_{0}$ only. For the intermediate and external domains, we choose instead

$$
X_{k j i}(\xi)=T_{i}(\xi)
$$

along with the collocation points 


$$
\xi_{i}=-\cos \left(\pi i /\left(N_{r}-1\right)\right) \quad 0 \leq i \leq N_{r}-1 .
$$

Note that for the nucleus the above choice is the same as that presented in [27], once $\xi$ is replaced by $r$. We report the interested reader to that paper for a more detailed discussion about this choice (see also Appendices A, B and D of 18 ).

When a symmetry is present, we use different bases, in order to take the symmetry into account. For instance, an often existing symmetry is the symmetry with respect to the equatorial plane, i.e. the plane $\theta=\pi / 2$. In this case, we use, instead of (73)-(74),

$$
\begin{aligned}
& \Theta_{k j}(\theta)=\cos (2 j \theta) \quad \text { for } m \text { even } \\
& \Theta_{k j}(\theta)=\sin ((2 j+1) \theta) \quad \text { for } m \text { odd } .
\end{aligned}
$$

The associated collocation points span only $[0, \pi / 2]$, instead of $[0, \pi]$ :

$$
\theta_{j}=\frac{\pi}{2} \frac{j}{N_{\theta}-1} \quad 0 \leq j \leq N_{\theta}-1 .
$$

Another usual symmetry is the above equatorial symmetry augmented by the symmetry under the transformation $\varphi \mapsto \varphi+\pi$. This is the case of a triaxial ellipsoid, or of an axisymmetric star perturbed by even $m$ modes. In this case, the $\varphi$-basis functions are

$$
\Phi_{k}(\varphi)=e^{2 i k \varphi} .
$$

The associated collocation points span $[0, \pi[$, instead of $[0,2 \pi[$ :

$$
\varphi_{k}=\pi k / N_{\varphi} \quad 0 \leq k \leq N_{\varphi}-1 .
$$

The basis in $\theta$ become

$$
\Theta_{k j}(\theta)=\cos (2 j \theta),
$$

instead of (81)-(82), the collocation points in $\theta$ remaining those given by Eq. (83). In this case, the basis for $\xi$ in the nucleus contain only even polynomials:

$$
X_{k j i}(\xi)=T_{2 i}(\xi),
$$

the collocation points remaining those given by Eq. (78).

\section{B. Differential operators}

In this section, we present how a first order differential operator, the gradient, and a second order one, the Laplacian, both applied to a scalar field, are expressed in term of the coordinates system described above. The computation of any other kind of operator is straightforward.

The components of the gradient of a scalar field $f$ in an orthonormal basis associated with the spherical coordinates $(r, \theta, \varphi)$ are

$$
\begin{aligned}
\frac{\partial f}{\partial r} & =J_{1}^{-1} \frac{\partial f}{\partial \xi} \\
\frac{1}{r} \frac{\partial f}{\partial \theta} & =\frac{1}{R_{l}} \frac{\partial f}{\partial \theta^{\prime}}-\frac{J_{2}}{J_{1}} \frac{\partial f}{\partial \xi} \\
\frac{1}{r \sin \theta} \frac{\partial f}{\partial \varphi} & =\frac{1}{R_{l} \sin \theta^{\prime}} \frac{\partial f}{\partial \varphi^{\prime}}-\frac{J_{3}}{J_{1}} \frac{\partial f}{\partial \xi}
\end{aligned}
$$

where the following abbreviations have been introduced:

$$
\begin{aligned}
J_{1} & :=\frac{\partial R_{l}}{\partial \xi} \\
J_{2} & :=\frac{1}{R_{l}} \frac{\partial R_{l}}{\partial \theta^{\prime}} \\
J_{3} & :=\frac{1}{R_{l} \sin \theta^{\prime}} \frac{\partial R_{l}}{\partial \varphi^{\prime}} .
\end{aligned}
$$


Note that we have re-introduced the primes on $\theta$ and $\varphi$ [cf. Eqs. (14)-(阿) to avoid any confusion between the partial derivatives. The partial derivatives that appear in the quantities $J_{i}$ are computed by (i) a (banded) matrix multiplication on the coefficients of the spectral expansion of the functions $F_{l}(\theta, \varphi)$ and $G_{l}(\theta, \varphi)$ and (ii) analytically for the polynomials $A_{l}(\xi)$ and $B_{l}(\xi)$. In the nucleus, $J_{2}$ is re-expressed as

$$
J_{2}=\frac{\left(3 \xi^{3}-2 \xi^{5}\right) \partial F_{0} / \partial \theta^{\prime}+\frac{1}{2}\left(5 \xi^{2}-3 \xi^{4}\right) \partial G_{0} / \partial \theta^{\prime}}{1+\left(3 \xi^{3}-2 \xi^{5}\right) F_{0}+\left(5 \xi^{2}-3 \xi^{4}\right) G_{0}}
$$

in order to avoid any division by a vanishing quantity at $\xi=0$. The same thing is done for $J_{3}$.

The above expressions are valid for the nucleus and the intermediate domains, i.e. for $l=0, \ldots, \mathcal{N}-2$. For the compactified domain $\mathcal{D}_{\text {ext }}$, the quantity to be considered is $r^{2} \nabla f$ instead $\nabla f$. Indeed, gradients in the compactified domain are used in the computation of non-linear terms in the relativistic gravitational field equations (scalar products of gradients of the metric potentials). We shall see below that the source of the Poisson equation on $\mathcal{D}_{\text {ext }}$ is to be multiplied by $r^{4}$, so that if each gradient is multiplied by $r^{2}$, this multiplication by $r^{4}$ is automatically performed. The orthonormal components of $r^{2} \nabla f$ on $\mathcal{D}_{\text {ext }}$ are

$$
\begin{aligned}
r^{2} \times \frac{\partial f}{\partial r} & =-\left(\frac{\partial U}{\partial \xi}\right)^{-1} \frac{\partial f}{\partial \xi} \\
r^{2} \times \frac{1}{r} \frac{\partial f}{\partial \theta} & =\frac{1}{U} \frac{\partial f}{\partial \theta^{\prime}}-\left(\frac{\partial U}{\partial \xi}\right)^{-1} \frac{1}{U} \frac{\partial U}{\partial \theta^{\prime}} \frac{\partial f}{\partial \xi} \\
r^{2} \times \frac{1}{r \sin \theta} \frac{\partial f}{\partial \varphi} & =\frac{1}{U \sin \theta^{\prime}} \frac{\partial f}{\partial \varphi^{\prime}}-\left(\frac{\partial U}{\partial \xi}\right)^{-1} \frac{1}{U \sin \theta^{\prime}} \frac{\partial U}{\partial \varphi^{\prime}} \frac{\partial f}{\partial \xi} .
\end{aligned}
$$

The Laplacian of a scalar field $f$ reads

$$
\begin{aligned}
\Delta f= & J_{1}^{-1}\left\{J_{1}^{-1}\left(1+J_{2}^{2}+J_{3}^{2}\right) \frac{\partial^{2} f}{\partial \xi^{2}}+\frac{2}{R_{l}} \frac{\partial f}{\partial \xi}\right\}+\frac{1}{R_{l}^{2}} \Delta_{\theta \varphi} f-J_{1}^{-1}\left\{2\left(\frac{J_{2}}{R_{l}} \frac{\partial^{2} f}{\partial \theta \partial \xi}+\frac{J_{3}}{R_{l} \sin \theta} \frac{\partial^{2} f}{\partial \varphi \partial \xi}\right)\right. \\
& \left.+\left[\frac{1}{R_{l}^{2}} \Delta_{\theta \varphi} R_{l}+J_{1}^{-1}\left(J_{1}^{-1}\left(1+J_{2}^{2}+J_{3}^{2}\right) \frac{\partial^{2} R_{l}}{\partial \xi^{2}}-2\left(\frac{J_{2}}{R_{l}} \frac{\partial^{2} R_{l}}{\partial \theta \partial \xi}+\frac{J_{3}}{R_{l} \sin \theta} \frac{\partial^{2} R_{l}}{\partial \varphi \partial \xi}\right)\right)\right] \frac{\partial f}{\partial \xi}\right\},
\end{aligned}
$$

where the primes on $\theta$ and $\varphi$ have been abandoned again and the following abbreviation has been introduced:

$$
\Delta_{\theta \varphi}:=\frac{\partial^{2}}{\partial \theta^{2}}+\frac{1}{\tan \theta} \frac{\partial}{\partial \theta}+\frac{1}{\sin ^{2} \theta} \frac{\partial^{2}}{\partial \varphi^{2}} .
$$

\section{Resolution of the Poisson equation}

For many astrophysical applications, one has to solve the Poisson-like equation

$$
\Delta f=\sigma(f),
$$

for some 'potential' $f$. Note that for relativistic computations, $\sigma(f)$ is not compactly supported (see e.g. [9]) and generally decreases as $1 / r^{4}$ when $r \rightarrow+\infty$.

When expressed in terms of the variables $(\xi, \theta, \varphi)$, the Laplacian takes the complicated form (98), for which it is not obvious to find eigenfunctions. Therefore, we introduce, in each domain $\mathcal{D}_{l}$, a new radial coordinate

$$
\zeta:=\alpha_{l} \xi+\beta_{l}
$$

where $\alpha_{l}$ and $\beta_{l}$ are the same constants as in Eqs. (11) and (34) (in the nucleus: $\beta_{0}=0$ ). In the exterior domain, we introduce

$$
\eta:=\alpha_{\text {ext }}(\xi-1),
$$

where $\alpha_{\text {ext }}$ is the same constant as in Eq. (62). We may then split the Laplacian operator $\Delta$ into a pseudo-Laplacian $\tilde{\Delta}$ and a part which would vanish if the domains $\mathcal{D}_{l}$ were exactly spherical (in this case, the coordinates $\zeta$ and $\eta$ introduced 
here above would coincide with the physical coordinates $r$ and $u=1 / r$ respectively). By pseudo-Laplacian, we mean the operator which once expressed in terms of $(\zeta, \theta, \varphi)$ has the same structure than the Laplacian operator in spherical coordinates:

$$
\tilde{\Delta}:=\frac{\partial^{2}}{\partial \zeta^{2}}+\frac{2}{\zeta} \frac{\partial}{\partial \zeta}+\frac{1}{\zeta^{2}} \Delta_{\theta \varphi}
$$

where $\Delta_{\theta \varphi}$ is defined by Eq. (99). In the exterior domain, the pseudo-Laplacian is defined instead by

$$
\tilde{\Delta}:=\frac{\partial^{2}}{\partial \eta^{2}}+\frac{1}{\eta^{2}} \Delta_{\theta \varphi} .
$$

It is much easier to invert the operator $\tilde{\Delta}$ than the operator $\Delta$ : using spherical harmonics in $(\theta, \varphi)$, the problem reduces to a system of second order ordinary differential equations with respect to the variable $\zeta$. Moreover, the junction conditions between the various domains are easily imposed, as explained below.

The Poisson equation (100) becomes

$$
a \tilde{\Delta} f=\sigma(f)+\mathcal{R}(f)
$$

where

$$
\begin{aligned}
& a:=\alpha_{l}^{2} J_{1}^{-2}\left(1+J_{2}^{2}+J_{3}^{2}\right) \\
& \mathcal{R}(f):= {\left[J_{1}^{-1} \frac{R_{l}}{\xi+\beta_{l} / \alpha_{l}}\left(1+J_{2}^{2}+J_{3}^{2}\right)-1\right] \frac{2}{J_{1} R_{l}} \frac{\partial f}{\partial \xi}+\left[J_{1}^{-2} \frac{R_{l}^{2}}{\left(\xi+\beta_{l} / \alpha_{l}\right)^{2}}\left(1+J_{2}^{2}+J_{3}^{2}\right)-1\right] \frac{1}{R_{l}^{2}} \Delta_{\theta \varphi} f } \\
&+J_{1}^{-1}\left\{2\left(\frac{J_{2}}{R_{l}} \frac{\partial^{2} f}{\partial \theta \partial \xi}+\frac{J_{3}}{R_{l} \sin \theta} \frac{\partial^{2} f}{\partial \varphi \partial \xi}\right)\right. \\
&\left.+\left[\frac{1}{R_{l}^{2}} \Delta_{\theta \varphi} R_{l}+J_{1}^{-1}\left(J_{1}^{-1}\left(1+J_{2}^{2}+J_{3}^{2}\right) \frac{\partial^{2} R_{l}}{\partial \xi^{2}}-2\left(\frac{J_{2}}{R_{l}} \frac{\partial^{2} R_{l}}{\partial \theta \partial \xi}+\frac{J_{3}}{R_{l} \sin \theta} \frac{\partial^{2} R_{l}}{\partial \varphi \partial \xi}\right)\right)\right] \frac{\partial f}{\partial \xi}\right\} .
\end{aligned}
$$

In order to let appear only the operator $\tilde{\Delta}$ in the left-hand-side of Eq. (105), we introduce

$$
a_{l}:=\max _{\mathcal{D}_{l}} a
$$

and recast Eq. (105) into

$$
\tilde{\Delta} f=\frac{1}{a_{l}}\left[\sigma(f)+\mathcal{R}(f)+\left(a_{l}-a\right) \tilde{\Delta} f\right] .
$$

Since $f$ appears on the right-hand-side of this equation, we solve it by iteration. Besides, we introduce some relaxation in the computation of the term $\tilde{\Delta} f$ in the right-hand-side of Eq. (109). More specifically, we solve at each step of the iterative scheme the following equation

$$
\tilde{\Delta} f^{J+1}=\tilde{\sigma}^{J}
$$

where the index $J$ denotes the step at which the quantities are taken and $\tilde{\sigma}^{J}$ is the following source, computed from the value of $f$ at the step $J$ :

$$
\tilde{\sigma}^{J}=a_{l}^{-1}\left\{\sigma\left(f^{J}\right)+\mathcal{R}\left(f^{J}\right)+\left(a_{l}-a\right)\left[\lambda \tilde{\sigma}^{J-1}+(1-\lambda) \tilde{\sigma}^{J-2}\right]\right\} .
$$

In this expression, $\lambda$ is a relaxation parameter (a typical value is $\lambda=1 / 2$ ) and $\mathcal{R}\left(f^{J}\right.$ ) is to be computed according to Eq. (107). For the first step $(J=0), f^{J}, \tilde{\sigma}^{J-1}$ and $\tilde{\sigma}^{J-2}$ are set to zero or to their value at a previous step in an evolutionary scheme.

We have exposed the method of resolution of Eq. (110) elsewhere 27,9]. Let us simply mention that we first perform a transformation from the bases in $(\theta, \varphi)$ described in Sect. III A (Chebyshev polynomials in $\cos \theta-$ Fourier expansion in $\varphi$ ) to spherical harmonics $Y_{\ell}^{m}(\theta, \varphi)$, by means of a matrix multiplication onto the coefficients of the $\theta$ expansion. For each value of $(\ell, m)$, Eq. (110) gives then a second order ordinary differential equation with respect to $\zeta$, the 
solution of which amounts to invert a banded matrix. Two solutions of the homogeneous equation $(\Delta f=0)$ are then added in order to connect the solution and its first derivative across the boundaries between the $\mathcal{N}$ domains. More precisely, the global boundary condition, generally $f \rightarrow 0$ when $r \rightarrow+\infty$, is imposed by setting the value of $f$ at the exterior boundary of the external domain, which is exactly $r=+\infty$ as explained in Sect. II D. The matching between the various domains amounts then to the resolution of a simple system of $2 \mathcal{N}-1$ linear equations for the coefficients of the homogeneous solutions to be added in each domain. Note that this matching is performed for each value of $(\ell, m)$.

\section{REGULARIZATION OF THE SOURCE OF POISSON EQUATION}

\section{A. Description of the method}

The analytical properties of the source of the gravitational field at the boundary of the star depend on the equation of state (EOS). For a polytrope of adiabatic index $\gamma\left(P \propto n^{\gamma}\right)$, the matter density $n$ behaves as $H^{1 /(\gamma-1)}$ where $H$ is the specific enthalpy. Consequently, for $\gamma>2$, the derivative $d n / d H$ has an infinite value for $H=0$, i.e. at the surface of the star and $d n / d r$ diverges at surface of the star. For values of $\gamma<2$ only derivatives of higher order diverge (actually there exists some value of $\gamma$, e.g. $\gamma=4 / 3$, for which all derivatives vanish or have a finite value at the surface of the star).

In a steady state configuration $H$ is Taylor expandable at the neighbourhood of the star's surface (this can be easily seen on Eq. (123) below). Therefore $H$ vanishes as $r-R(\theta, \varphi)$ where $R(\theta, \varphi)$ is the equation of the star's surface and $n$ behaves as $n \sim[r-R(\theta, \varphi)]^{1 /(\gamma-1)}$ (this analysis remains valid even for EOS more general than the polytropic one if $\gamma$ is defined as $\gamma=d\left(\ln (P) /\left.d \ln (H)\right|_{H=0}\right)$. Consequently $n$ is generaly not a $\mathcal{C}^{\infty}$ function. This singular behaviour implies that the $\mathcal{L}^{2}$ truncation error of the spectral approximation is no more evanescent and moreover that Gibbs phenomenon is present. This fact is especialy awkward when studying the stability of equilibrium configurations or looking for bifurcation points because high accuracy is required. In practice $\gamma$ cannot be larger than 3 [16, 17. Note that in the litterature the potential in spherical coordinates is often computed by expanding the source in spherical harmonics $Y_{l}^{m}(\theta, \varphi)$ and by computing the radial part with a finite difference method. In this case the Gibbs phenomenon will appear in the angular part of the solution. The situation is even worse if the radial part of the potential is computed with a spectral method. A method to recover spectral accuracy in such cases is as follows.

We first introduce a known potential $\Phi_{\text {div }}$ such that $n_{\text {div }}:=\Delta \Phi_{\text {div }}$ as the same pathological behaviour as $n$ and such that $n-n_{\text {div }}$ is a regular function (at least more regular then $n$ ) and numericaly solve

$$
\Delta \Phi_{\text {regu }}=n-n_{\text {div }}
$$

where $\Phi_{\text {regu }}:=\Phi-\Phi_{\text {div }}$.

Consider for instance

$$
\Phi_{\mathrm{div}}=F(\xi, \theta, \varphi)\left(1-\xi^{2}\right)^{(\alpha+2)}
$$

where $\alpha=1 /(\gamma-1), F$ is an arbitrary regular function and $\xi$ is a new radial variable such that $\xi=1$ at the surface of the star (see Sect. III). It is easy to see that $\Delta \Phi_{\text {div }}$ has a term vanishing at the surface as $(1-\xi)^{\alpha}$ (i.e. with the same pathological behaviour as $n$ ). We have indeed

$$
\begin{aligned}
\tilde{\Delta} \Phi_{\mathrm{div}}= & \tilde{\Delta} F \xi^{2}\left(1-\xi^{2}\right)^{(\alpha+2)}-4(\alpha+2) \xi\left(1-\xi^{2}\right)^{(\alpha+1)} \partial_{\xi} F \\
& +(\alpha+2)\left[-6\left(1-\xi^{2}\right)^{(\alpha+1)}+4(\alpha+1) \xi^{2}\left(1-\xi^{2}\right)^{\alpha}\right] F
\end{aligned}
$$

where $\tilde{\Delta}$ is the Laplacian computed with respect to $(\xi, \theta, \varphi)$ (cf. Eq. (103)).

The choice of the factor of $(1-\xi)^{(\alpha+2)}$ is done in order that $\Phi_{\text {div }}$ has the required regularity properties at $\xi=0$ and the required behaviour at the boundary of the star. The choice of $F(\xi, \theta, \varphi)$ is arbitrary. If we choose for $F(\xi, \theta, \varphi)$ an harmonic function, $\tilde{\Delta} F=0$, the first term of the r.h.s. of the Eq. (114) vanishes. This is an advantage because this term can be quite large and, consequently, give rise to a large error in computing $\Phi_{\text {regu }}$. We write $\Phi_{\text {div }}=\sum_{l, m} a_{l m} \Phi_{l m}$, where

$$
\Phi_{l m}:=\xi^{l}\left(1-\xi^{2}\right)^{(\alpha+2)} Y_{l}^{m}(\theta, \varphi)
$$

and where $a_{l m}$ are some numerical coefficients to be determined. We then obtain $n_{\text {div }}(\xi, \theta, \varphi)=$ $\sum_{l, m} a_{l m} C_{l}(\xi) Y_{l}^{m}(\theta, \varphi)$, with 


$$
C_{l}(\xi):=(\alpha+2)\left[-(4 l+6)\left(1-\xi^{2}\right)^{(\alpha+1)} \xi^{l}+4(\alpha+1) \xi^{(l+2)}\left(1-\xi^{2}\right)^{\alpha}\right] .
$$

We have now to determine the values of the coefficients $a_{l m}$ which give the most regular function $n_{\text {regu }}:=n-n_{\text {div }}$. The criterion which seems to give the best results is the following one.

We expand $n$ and $n_{\text {div }}$ as truncated series of spherical harmonics $Y_{l}^{m}(\theta, \varphi)$ and Chebyshev polynomial $T_{i}(\xi)$ :

$$
n(\xi, \theta, \varphi)=\sum_{i, l, m=0}^{I, L, M} n_{i l m} T_{i}(\xi) Y_{l}^{m}(\theta, \varphi)
$$

and each of the functions $C_{l}(\xi)$ in a Chebyshev series:

$$
C_{l}(\xi)=\sum_{i}^{I} C_{l i} T_{i}(\xi)
$$

The value of $a_{l m}$ is computed in such a way that the $I^{\text {th }}$ coefficient of the truncated series of $n_{\text {regu vanishes: }}$

$$
a_{l m}=n_{I l m} / C_{l I}
$$

By means of the above procedure, we eliminate in $n$ the pathological term vanishing as $(1-\xi)^{\alpha}$ but we introduce another pathological term $\propto\left(1-\xi^{2}\right)^{\alpha+1}$. However the divergence occurs in a higher order derivative of this term so that it has a much weaker effect on the accuracy of the result. The method can be improved by taking

$$
\Phi_{\mathrm{div}}=F(\xi, \theta, \varphi)\left(1-\xi^{2}\right)^{\alpha+2}\left[a_{1}+a_{2}\left(1-\xi^{2}\right)+a_{3}\left(1-\xi^{2}\right)^{2}+\cdots+a_{K}\left(1-\xi^{2}\right)^{K-1}\right] .
$$

instead of (113). The coefficients $a_{k}$ are chosen in a such a way that the $1^{\text {st }}, 2^{t h}, \ldots, K^{t h}$ derivatives of $n_{\text {regu }}$ vanish at $\xi=1$. Let us call $K$ the regularization degree of the procedure.

Note that, since $\Phi_{\text {div }}$ and $\partial_{\xi} \Phi_{\text {div }}$ vanish at the surface of the star, the boundary condition one has to impose to solve $\Delta \Phi_{\text {regu }}=n_{\text {regu }}$ is the same than that for $\Delta \Phi=n$. We want to point out that, the above regularization technique can be used, mutatis mutandis, also when a finite difference method is used.

\section{B. Examples}

Consider two polytropic EOS of adiabatic index $\gamma=3$ and $\gamma=10$ with a spherically symmetric distribution of the enthalpy $H=1-\xi^{2}$. The corresponding sources density are $n_{3}(\xi)=\left(1-\xi^{2}\right)^{1 / 2}$ and $n_{10}(\xi)=\left(1-\xi^{2}\right)^{1 / 9}$. Figure 2 shows the mass distributions $n$ and $n_{\text {regu }}$ for various values of the regularization degree $K$ [Eq. (120)]. Note that in the case of $\gamma=10$ the procedure improves considerably the behaviour of the source $n_{\text {regu }}$ even for $K=1$.

$$
\gamma=3
$$

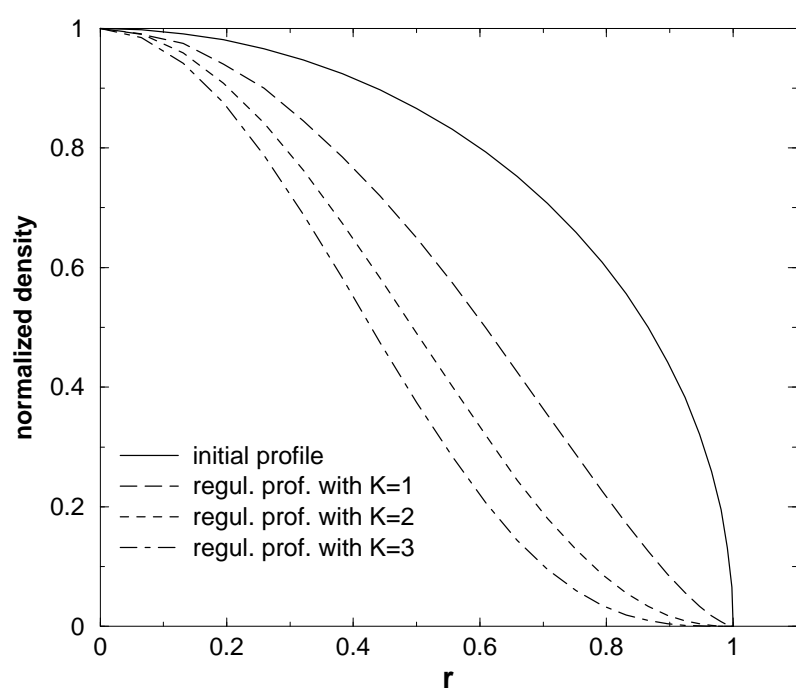

$\gamma=10$

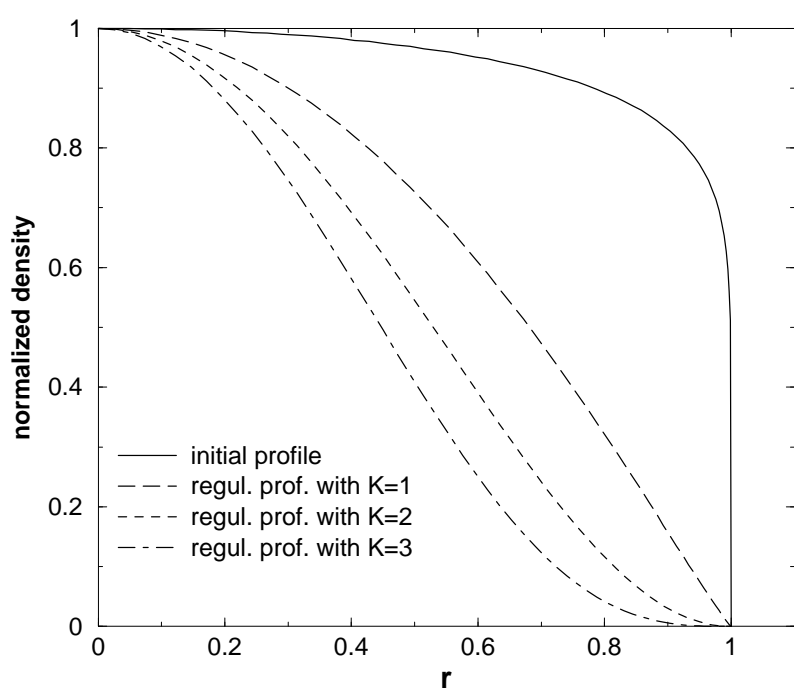

FIG. 2. Original and regularized density profiles for $\gamma=3$ and $\gamma=10$ polytropes. The regularized profiled are rescaled to take the value 1 at the origin. 
The method can be tested in the case of $\gamma=3$ by direct comparison with the analytical solution. In this case the gravitational field $G=\partial_{r} \Phi$ reads

$$
G=\frac{1}{r^{2}} \int_{0}^{r}\left(1-u^{2}\right)^{1 / 2} u^{2} d u=\frac{1}{8 r^{2}}\left[\arcsin r+r\left(1-r^{2}\right)^{1 / 2}-2 r\left(1-r^{2}\right)^{3 / 2}\right]
$$

Figure 3 shows the relative $\mathcal{L}^{1}$ error $\epsilon$ on $G$ as a function of the number of degrees of freedom $N_{r}$. The error $\epsilon$ follows approximately a power law $\epsilon \propto N_{r}^{-\beta}$. The dependence of the exponent $\beta$ with respect to the regularization degree $K$ is shown in Figure 4 . A value as high as of $\beta \approx 17$ can be achieved with only $K=6$. Note that the relation $\epsilon=N_{r}^{-\beta}$ is only an approximate law. This means that the error tends to become evanescent when the regularization degree increases.

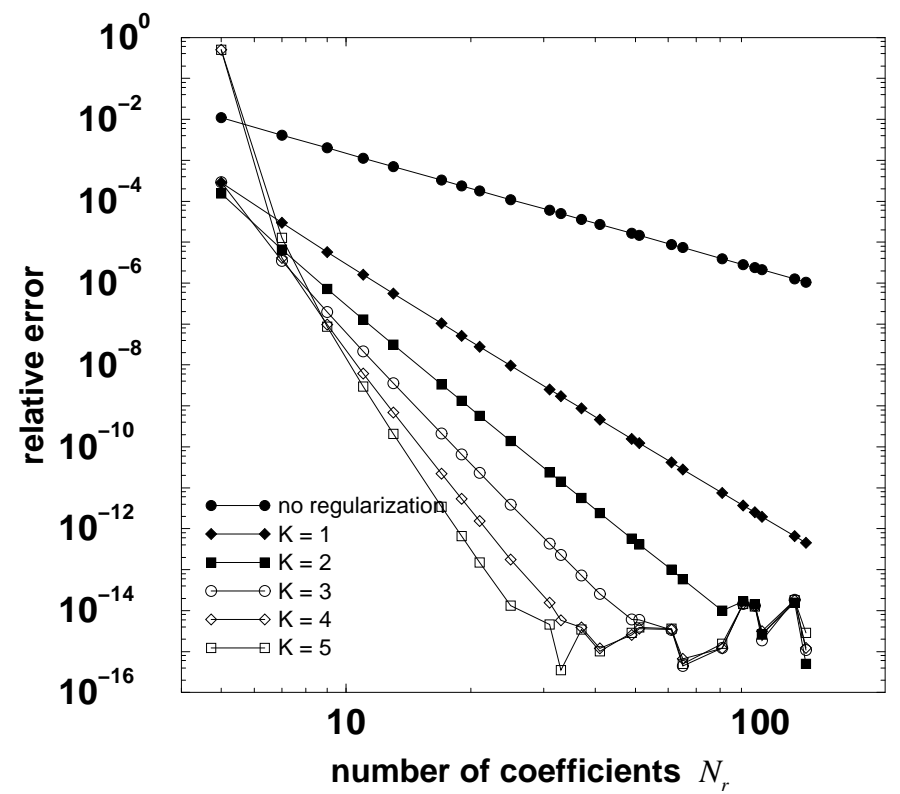

FIG. 3. Relative $\mathcal{L}^{1}$ error $\epsilon$ on the gravitational field as a function of the number of degrees of freedom $N_{r}$ for different regularization degrees $K$.

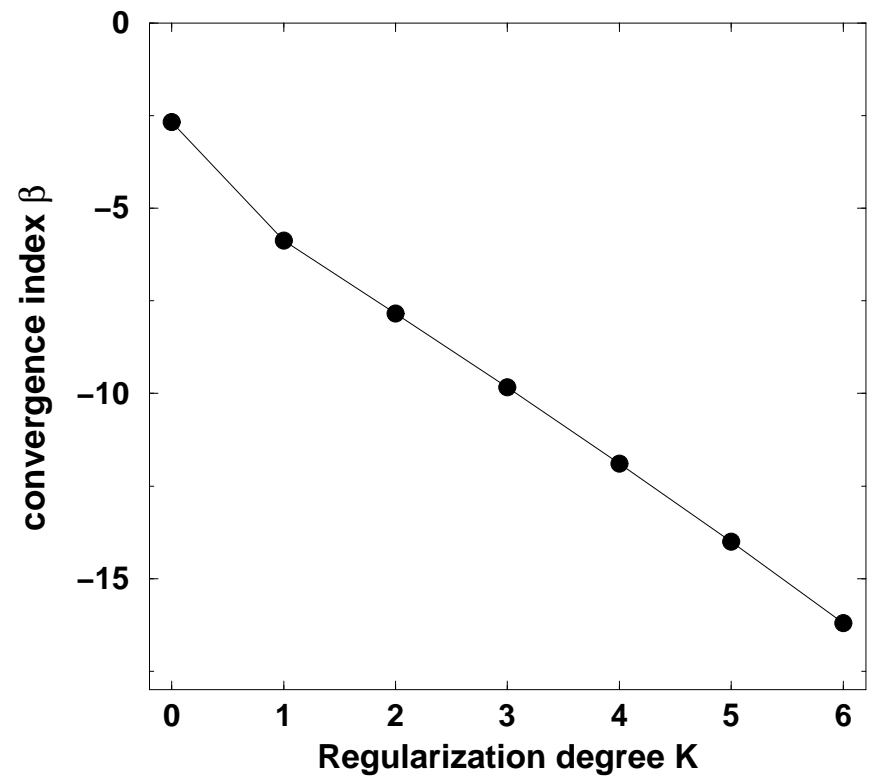

FIG. 4. Dependence of the exponent $\beta$ on the regularization degree $K$. 


\section{ILLUSTRATIVE APPLICATIONS}

\section{A. 3-D stationary configurations}

In this section, we sketch the general structure of a code for computing single star stationary configurations under the influence of rotation and a tidal potential. For simplicity we present only the Newtonian case, the relativistic one showing no new qualitative feature but simply involving more equations.

The equilibrium configuration of a cold star rotating rigidly at the angular velocity $\Omega$ with respect to some inertial frame and embedded in a tidal potential $\Phi_{\text {tide }}$ is governed by the following three equations ${ }^{\mathbb{T}}$

$$
\begin{gathered}
\Delta \Phi_{\text {grav }}=4 \pi G \rho, \\
H+\Phi_{\text {grav }}-\frac{1}{2}(\Omega r \sin \theta)^{2}+\Phi_{\text {tide }}=\text { const } \\
\rho=\rho(H) .
\end{gathered}
$$

Equation 122 is the Poisson equation linking the gravitational potential $\Phi_{\text {grav }}$ to the mass density $\rho$. Equation (123) is the first integral which can be derived from the Euler equation governing the (perfect) fluid velocity under the stationarity assumption; this equation relates the specific enthalpy of the fluid $H$ to the internal and external potentials. Finally Eq. (124) is the matter equation of state in the zero-temperature approximation.

The number of domains used for solving this problem is $\mathcal{N}=3$ : one domain $\mathcal{D}_{0}$ for the star (the nucleus, cf. Sect. IIB), one intermediate domain $\mathcal{D}_{1}$ (cf. Sect. IIC), the outer boundary of which is spherical and the external domain $\mathcal{D}_{2}$ (cf. Sect. IID). In fact, if one would have to treat only the Newtonian case, one domain would be sufficient (i.e. the nucleus) because Eq. (122) has a compact support, which is no more true in the relativistic case.

A solution is specified by the central value of $H$ (or $\rho$ ), $H_{\mathrm{c}}$ say, the value of $\Omega$ and the expression of $\Phi_{\text {tide }}$. These quantities being given, the iterative method of resolution is as follows. The $\mathcal{N}$ domains are first taken to be exactly spherical. One starts from a very crude density profile, for instance $\rho=$ const in $\mathcal{D}_{0}$. Solving the Poisson equation $(122)$ by means of the method presented in Sect. III leads to the gravitational potential $\Phi_{\text {grav }}$. Inserting its value into Eq. (123) give a new profile for the specific enthalpy $H$ (the constant on the right-hand-side of Eq. (123) is fully determined by the requirement that $H=H_{\mathrm{c}}$ at the centre of the star). The surface of the star being defined by $H=0$, its equation $r=S_{0}(\theta, \varphi)$ [using the notation of Eq. (11)] is found by searching for the equipotential $H=0$ in the newly determined $H$ field. This defines a new domain $\mathcal{D}_{0}$. The corresponding mapping $R_{0}(\xi, \theta, \varphi)$, i.e. the value of the constant $\alpha_{0}$ and the functions $F_{0}(\theta, \varphi)$ and $G_{0}(\theta, \varphi)$ [cf. Eq. (11)] is computed according to the procedure described in Sect. II B. The new intermediate domain $\mathcal{D}_{1}$ is defined by the new inner boundary $\mathcal{S}_{0}$ (the surface of the star) and the unchanged spherical outer boundary $\mathcal{S}_{1}$. The corresponding mapping $R_{1}(\xi, \theta, \varphi)$ is computed according to the procedure described in Sect. II C. The external domain $\mathcal{D}_{2}$ remains unchanged.

The physical location $(r, \theta, \varphi)$ of the collocation points $\left(l, \xi_{i}, \theta_{j}, \varphi_{k}\right)$ (where $l$ is the domain index) corresponding to these new mappings is a priori different from that of the previous mappings, where all the fields were known. Therefore, one has to compute the values of the fields at the new collocation points. In the present case, it is sufficient to do so only for the specific enthalpy $H$. In the domain no. $l$, the collocation point $\left(\xi_{i}, \theta_{j}, \varphi_{k}\right)$ has the physical radial coordinate

$$
r=R_{l}^{J}\left(\xi_{i}, \theta_{j}, \varphi_{k}\right)
$$

where the superscript $J$ refers to the step in the iterative procedure: $R_{l}^{J}(\xi, \theta, \varphi)$ is the current value of the mapping of the domain $\mathcal{D}_{l}$, whereas $R_{l}^{J-1}(\xi, \theta, \varphi)$ is the previous value. Let us denote by $\left(L^{J-1}(r, \theta, \varphi), \Xi^{J-1}(r, \theta, \varphi)\right)$ the inverse mapping at the previous step. This inverse mapping is computed by searching for the zero of the function $(l, \xi) \mapsto r-R_{l}(\xi, \theta, \varphi)$. The values of $H$ at the collocation points of the new mapping are given by

$$
H^{J}\left(l, \xi_{i}, \theta_{j}, \varphi_{k}\right)=H^{J-1}\left(L^{J-1}\left(r^{J}, \theta_{j}, \varphi_{k}\right), \Xi^{J-1}\left(r^{J}, \theta_{j}, \varphi_{k}\right), \theta_{j}, \varphi_{k}\right),
$$

${ }^{1}$ see [28] for a discussion of these equations, including the relativistic case. 
where $r^{J}:=R_{l}^{J}\left(\xi_{i}, \theta_{j}, \varphi_{k}\right)$. The value of $H$ on the right-hand-side is to be taken at a point which a priori does not coincide with a collocation point in $\xi$. It is computed by a direct summation, by means the spectral expansion of $H$. Using the notations of Sect. III A, it writes

$$
H(l, \xi, \theta, \varphi)=\sum_{k=0}^{N_{\varphi}-1}\left[\sum_{j=0}^{N_{\theta}-1}\left(\sum_{i=0}^{N_{r}-1} \hat{H}_{l k j i} X_{k j i}(\xi)\right) \Theta_{k j}(\theta)\right] \Phi_{k}(\varphi)
$$

where the $\hat{H}_{l k j i}$ are the coefficients of $H$ is the domain no. $l$. Note that from the computational point of view, this summation is the most expensive operation of the method: it scales indeed as $\left(N_{r} N_{\theta} N_{\varphi}\right)^{2}$. It may be possible to replace the whole summation (127) by a truncated one or by some interpolation from the values of $H$ at the collocation points, in order to reduce the computational cost. The main advantage of the summation (127) is that it does not introduced any additional error in the method: the right-hand-side of Eq. (127) is the value of $H$ at the specified point within the spectral accuracy.

Once $H$ is computed at the collocation points of the new mapping by means of Eq. (126), the equation of state (124) is used to find the values of the mass density $\rho$ at the collocation points. A new iteration may then begin.

In all the computations we have made, we have found that this procedure converges. For stationary rotating stars in general relativity, a rigorous proof of convergence of such iterative method (except for the re-mapping of the physical space at each step) has been given by Schaudt \& Pfister [29].

\section{B. MacLaurin ellipsoids}

The multi-domain spectral method can handle constant density (incompressible matter) rotating bodies without any Gibbs phenomenon. With classical spectral methods, the Gibbs phenomenon would have been very severe since the density itself, and not some of its derivatives, is discontinuous across the stellar surface for incompressible fluids. This gives us the opportunity to quantify the accuracy of the method since exact analytical solutions are known for incompressible bodies: the so-called ellipsoidal figures of equilibrium (see e.g. [30]). Note that an ellipsoid is not a particular case for the mapping (11): all the coefficients of the expansion of $F_{0}(\theta, \varphi)$ and $G_{0}(\theta, \varphi)$ onto the bases described in Sect. III A are non-zero. In this respect, the ellipsoidal figures constitute a strong test of the method.

\section{MacLaurin ellipsoid}

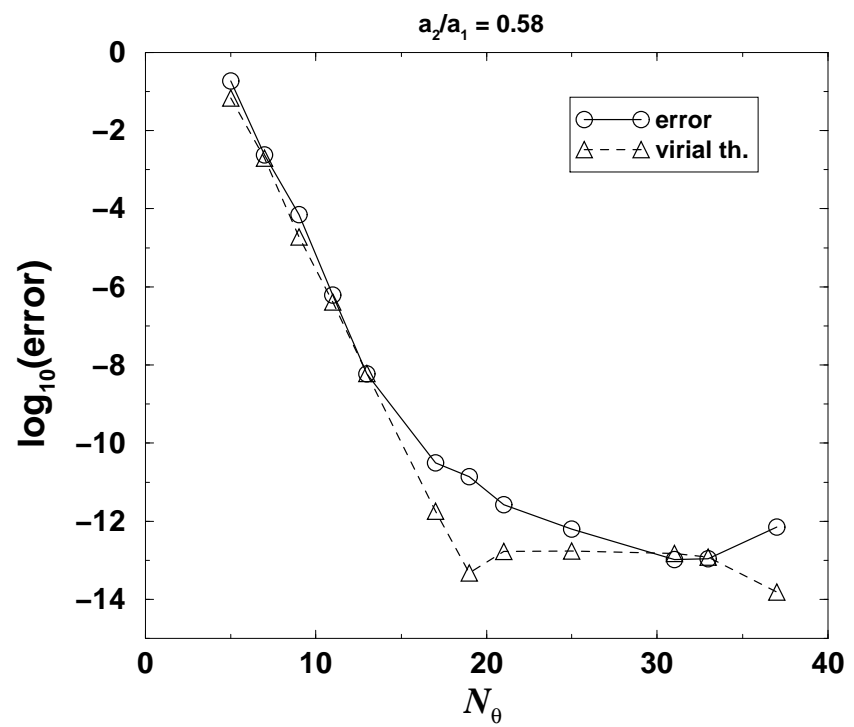

FIG. 5. Logarithm of the relative error of the numerical solution with respect to the number of degrees of freedom in $\theta$ for a MacLaurin spheroid at the Jacobi-Dedekind bifurcation point (the number of degrees of freedom in $r$ is $N_{r}=2 N_{\theta}-1$ ). Also shown is the error in the verification of the virial theorem.

For single rigidly rotating objects in the Newtonian regime, the more simple ellipsoidal solutions are constituted by the family of MacLaurin spheroids, which are axisymmetric about their rotation axis. We have computed them by means of the procedure presented in Sect. $\mathrm{VA}$, setting $\Phi_{\text {tide }}=0$ and the equation of state (124) to be simply 
$\rho=$ const. The axisymmetry allows to employ $N_{\varphi}=1$. The code converges towards ellipsoidal configurations and we measure the error by comparing the eccentricity $e:=\sqrt{1-\left(r_{\mathrm{p}} / r_{\mathrm{eq}}\right)^{2}}$ (where $r_{p}$ and $r_{\mathrm{eq}}$ are respectively the polar and equatorial radii) of the numerical solution with that of the analytical solution. The result of this comparison is presented in Fig. f for a MacLaurin spheroid located on the MacLaurin sequence at the point where the Jacobi and Dedekind sequences branch off: the eccentricity is $e=0.8127$, which corresponds to the ratio $r_{\mathrm{p}} / r_{\mathrm{eq}}=0.5827$. Shown in Fig. 5 is the relative error on the eccentricity as a function of the number of coefficients in the $\theta$ expansion. For these calculations, the number of coefficients in the $\xi$ expansion in each domain is $N_{r}=2 N_{\theta}-1$. The straight line behaviour of the left side of Fig. . shows that the error is evanescent, i.e. that it decreases as $\exp \left(-N_{\theta}\right)$. For $N_{\theta} \gtrsim 20$, the error saturates at the level of $10^{-12}-10^{-11}$. This is due to the round-off errors in the computation, which is performed with a 15-digit accuracy. It is instructive to compare this result with that obtained with a classical spectral method, i.e. with fixed spherical domains, as exposed in [9]. For instance, the Fig. 5 can be directly compared with Fig. 7 of [9]: this latter shows an power-law error decay only (of the type $N_{\theta}^{-4.5}$ ), due to the Gibbs phenomenon at the star's surface. Moreover, the error saturates at the level of $10^{-5}$. Note that this result was obtained with a polytropic equation of state (adiabatic index $\gamma=2$ ), for which the density is continuous across the surface of star; the fixed-spherical-domain spectral method presented in [9] was not able to treat incompressible fluid.

Also shown in Fig. f is the relative accuracy with which the 3-D virial theorem is satisfied. The 3-D Newtonian virial theorem 2 states that for a stationary configuration $2 T+3 P+W=0$, where $T$ is the total kinetic energy (with respect to the inertial frame), $P$ is the integral of the pressure throughout the star and $W$ is the gravitational potential energy. We have computed each of these three integrals for the numerical solution and evaluated the quantity

$$
\varepsilon:=\left|1-\frac{2 T+3 P}{|W|}\right| .
$$

For an exact solution, $\varepsilon=0$. The triangles plotted in Fig. 同 depict the value of $\log _{10} \varepsilon$ for the numerical models. Figure a shows that the virial error is very well correlated with the error evaluated by a direct comparison with the analytical solution. This gives us a strong confidence when using the virial error to evaluate the numerical error in more general cases, when no analytical solution is available.

\section{Roche ellipsoids}

Roche ellipsoids are equilibrium solutions for incompressible fluid bodies in a synchronized binary system, within the approximation of taking only the second order term in the expansion (around the center of mass of one star) of the gravitational potential of the companion. They are obtained by setting

$$
\Phi_{\text {tide }}=-\frac{G M_{\text {comp }}}{|a|}\left(1+\frac{x}{a}+\frac{2 x^{2}-y^{2}-z^{2}}{a^{2}}\right)
$$

in Eq. (123), where $a$ is the abscissa of the center of mass of the companion in the Cartesian frame $(x, y, z)$ centered at the center of mass of the star under consideration. Note that in Eq. (123), $r$ must now be the distance to the center of mass of the binary system and $\theta$ the angle with respect to the rotation axis of the system. Moreover, $\Omega$ must be chosen so that $\Omega^{2}=G\left(M+M_{\text {comp }}\right) / a^{3}$, in order that the linear term in $x$ which appear in Eq. (123) vanishes and one is left with an ellipsoidal solution.

The analytical solutions for Roche ellipsoid are given in the classical book by Chandrasekhar [30]. However they are given with an accuracy of five digit only (Table XVI in Ref. [30]), which is not sufficient for our comparison project: the accuracy achieved by the numerical code is far better than $10^{-5}$ as we shall see below. Therefore, we have written a small Mathematica [33] program to compute Chandrasekhar's "index symbol" $A_{1}, A_{2}$ and $A_{3}$ and obtain Roche solutions with an arbitrary number of digits.

Figure 6 present the results of the comparison between the numerical solution obtained by means of the method described in Sect. $\mathrm{VA}$ and the analytical solution. Let us recall that ellipsoidal shapes are not privileged in our formalism, so that this type of comparison constitute a strong test of our method. The comparison is conducted at fixed values of $\Omega / \rho$ and the mass ratio $M_{\text {comp }} / M$. Two global errors can be then defined: (i) the error on the axis ratio $a_{2} / a_{1}$ and (ii) the error on the axis ratio $a_{3} / a_{1}, a_{1}$ being the major axis of the triaxial ellipsoid (directed along the line of the two centers of mass), $a_{2}$ being the orthogonal axis in the orbital plane, and $a_{3}$ being the axis perpendicular

${ }^{2}$ as opposed to the 2-D virial identity, see 31] and 32 for a discussion 
to the orbital plane. These two errors are shown in Figure 6 for a Roche ellipsoid with $\Omega^{2} /(\pi G \rho)=0.1147$ and $M_{\text {comp }} / M=1$. The corresponding axis ratios are $a_{2} / a_{1}=0.7506$ and $a_{3} / a_{1}=0.6853$. The numerical solution is depicted in Fig. . 7 by three plane sections obtained with the following (small) numbers of coefficients: $N_{r}=13, N_{\theta}=7$ and $N_{\varphi}=6$. Also show in this Figure is the numerical grid (collocation points) used in the problem (only the domain $\mathcal{D}_{0}$ and a part of $\mathcal{D}_{1}$ are represented in the Figure). Even with such a small number of points, the relative error is of order $1 \times 10^{-4}$ (cf. Fig. 6) ! This explains why despite the numerical grid is quite coarse, the iso-enthalpy surfaces shown in Fig. 7 are so smooth.

Figure f gives the two global errors as a functions of the number of coefficients in the $\theta$ expansions, $N_{\theta}$. The number of coefficients employed in the other directions are $N_{r}=2 N_{\theta}-1$ and $N_{\varphi}=N_{\theta}-1$. As in Fig. f, the exponential decay of the error for $N_{\theta} \lesssim 13$ means that the error is evanescent. For $N_{\theta} \gtrsim 19$, the error saturates at the level of a few $10^{-10}$ due to the round-off errors in the computation, this latter being performed with a 15-digit accuracy. The

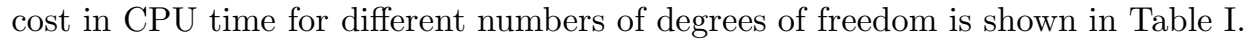

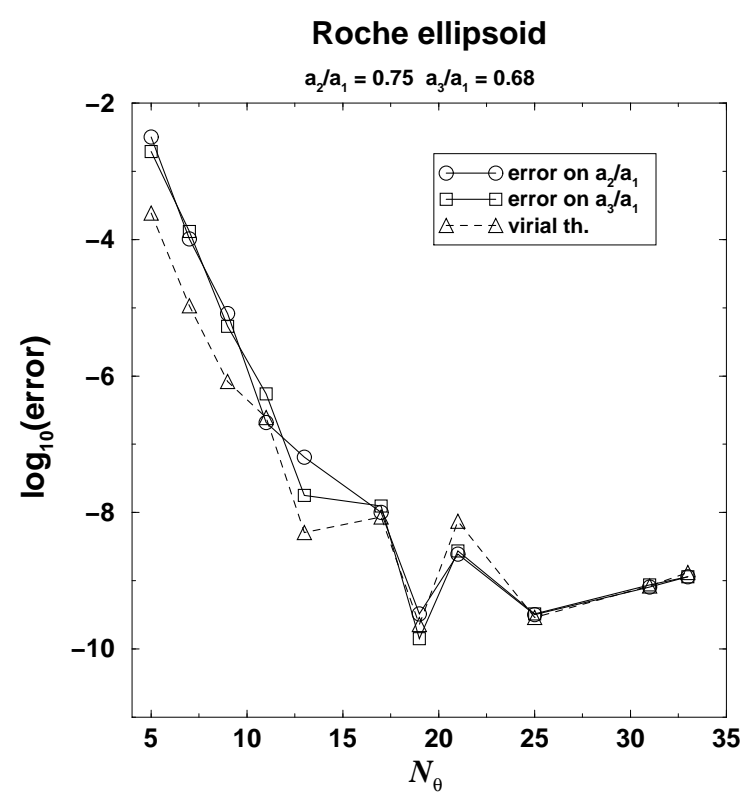

FIG. 6. Logarithm of the relative global error of the numerical solution with respect to the number of degrees of freedom in $\theta$ for a Roche ellipsoid for an equal mass binary system and $\Omega^{2} /(\pi G \rho)=0.1147$ (the numbers of degrees of freedom in the other directions are $N_{r}=2 N_{\theta}-1$ and $\left.N_{\varphi}=N_{\theta}-1\right)$. Also shown is the error in the verification of the virial theorem.

TABLE I. CPU time cost on a R4400/150MHz processor as a function of the number of degrees of freedom for the calculation of the Roche ellipsoid configuration corresponding to Fig. 6. The iteration is halted when the relative discrepancy between two successive steps reaches $10^{-13}$.

\begin{tabular}{lcccc}
\hline \hline$N_{r}$ & $N_{\theta}$ & $N_{\varphi}$ & \# of steps & CPU time per step (s) \\
\hline 25 & 13 & 12 & 116 & 6.92 \\
33 & 17 & 16 & 107 & 24.2 \\
49 & 25 & 24 & 115 & 189.16 \\
65 & 33 & 32 & 106 & 861.6 \\
\hline \hline
\end{tabular}



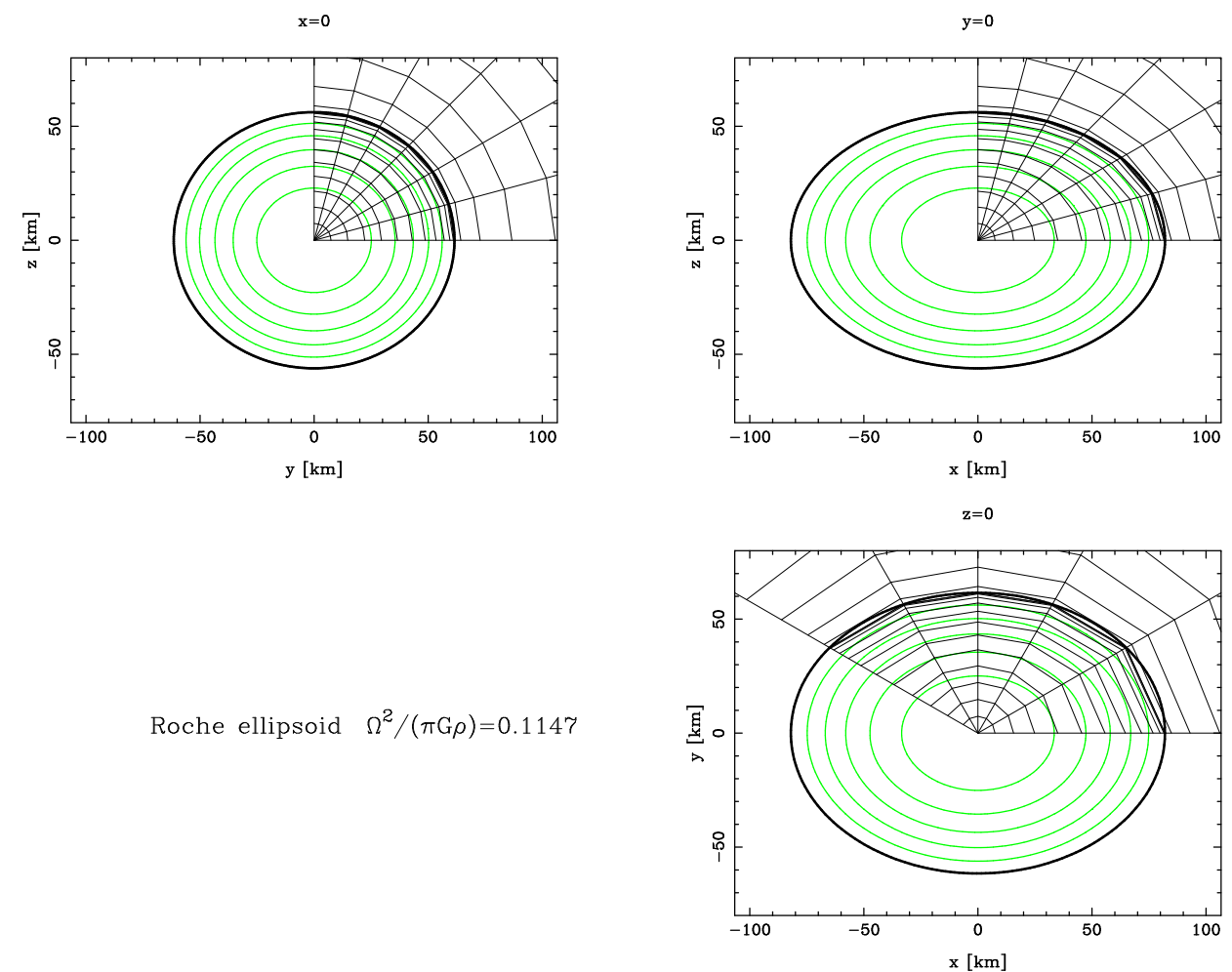

FIG. 7. Orthogonal plane sections in the numerical solution obtained for the Roche ellipsoid represented by the second set of points starting from the left on Fig. 6 (i.e. corresponding to $N_{r}=13, N_{\theta}=7$ and $N_{\varphi}=6$ ). Shown are the iso-enthalpy lines, as well as the numerical grid. This computation took a few seconds on a R4400/150MHz processor.

Also shown in Fig. 6 is the relative accuracy with which the 3-D virial theorem is satisfied. This error estimator is defined in the same way as in Sect. VB. As in the axisymmetric case (MacLaurin ellipsoids), we find a high correlation between the virial error and the errors obtained by direct comparison with the analytical solution.

\section{CONCLUSION AND PERSPECTIVES}

We have presented a new numerical approach capable of handling the surface discontinuities of stellar configurations, provided these discontinuities are star-like, which covers a wide range of astrophysically relevant situations. When used along with spectral methods this adaptive-domain technique ensures that no Gibbs phenomenon can appear. This results in a very high precision (evanescent error), as demonstrated in Sect. V by comparison with exact analytical solutions. The relative error for 3-D configurations can reach $10^{-10}$ with a relatively small number of degrees of freedom $\left(N_{r} \times N_{\theta} \times N_{\varphi}=37 \times 19 \times 18\right.$ in each domain). Let us recall that very high accuracy is required for a lot of astrophysical problems such as numerical stability analysis. Among these problems let us mention the study of symmetry breaking of rapidly rotating stars and the determination of the orbital frequency of the last stable orbit of a neutron stars binary system.

The multi-domain spectral method is particularly well adapted to the computation of relativistic binary neutron star system. Three sets of domains can be used in this problem (see Fig. 8): two sets of (three or more) domains centered on each star and a third set of (two or more) domains centered at the intersection between the rotation axis and the orbital plane. This latter set of domains which reaches spatial infinity is required to compute the gravitational field of relativistic configurations. When needed, the quantities computed on one of the three domain sets are evaluated at the collocation points of another set by means of the method presented in Sect. VA. We are currently applying this numerical method to the computation of steady-state configurations of relativistic counter-rotating (i.e. irrotational with respect to an inertial frame) neutron star binaries, following the formulation developed in [1]. We will report on the astrophysical results in a forthcoming paper.

An interesting byproduct of the present technical paper is the following one. In a previous work [9], we had been able to demonstrate that the virial error is representative of the true error (measured by direct comparison with analytical solutions) only in the spherically symmetric case. We had inferred that this remains valid in the axisymmetric and 3 -D cases. In the present work, we have confirmed this conjecture, thanks to the ability of the present method to 
treat incompressible fluids, for which 3-D analytical solutions are available.

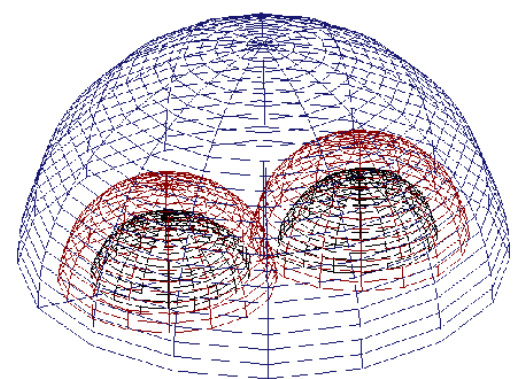

FIG. 8. Representation of the numerical domains that we use to compute relativistic steady-state configurations of binary neutron stars systems. The external domain extends to spatial infinity in order to compute the exact gravitational potentials. Due to the symmetry of the problem, only the $z>0$ part of space is taken into account.

[1] S. Bonazzola, E. Gourgoulhon, and J-A. Marck, Phys. Rev. D 56, 7740 (1997).

[2] S. Bonazzola, E. Gourgoulhon, and J-A. Marck, in Relativistic Gravitation and Gravitational Radiation, edited by JA. Marck and J.-P. Lasota (Cambridge University Press, Cambridge, England, 1997).

[3] S. Bonazzola and J-A. Marck, Astron. Astrophys. 267, 623 (1993).

[4] E. Gourgoulhon, Astron. Astrophys. 252, 651 (1991).

[5] E. Gourgoulhon and P. Haensel, Astron. Astrophys. 271, 187 (1993).

[6] E. Gourgoulhon, P. Haensel, and D. Gondek, Astron. Astrophys. 294, 747 (1995).

[7] J. Novak J., Phys. Rev. D 57, 4789 (1998).

[8] J-A. Marck, S. Bonazzola, and A. Lioure, Astron. Astrophys. 306, 666 (1996).

[9] S. Bonazzola, E. Gourgoulhon, M. Salgado, and J-A. Marck, Astron. Astrophys. 278, 421 (1993).

[10] M. Salgado, S. Bonazzola, E. Gourgoulhon, and P. Haensel, Astron. Astrophys. 291, 155 (1994).

[11] M. Salgado, S. Bonazzola, E. Gourgoulhon, and P. Haensel, Astron. Astrophys. suppl. 108, 455 (1994).

[12] P. Haensel, S. Bonazzola, and M. Salgado, Astron. Astrophys. 296, 745 (1995).

[13] M. Bocquet, S. Bonazzola, E. Gourgoulhon, and J. Novak, Astron. Astrophys. 301, 757 (1995).

[14] P. Haensel and S. Bonazzola, Astron. Astrophys. 314, 1017 (1996).

[15] S. Bonazzola and E. Gourgoulhon, Astron. Astrophys. 312, 675 (1996).

[16] S. Bonazzola, J. Frieben, and E. Gourgoulhon, Astrophys. J. 460, 379 (1996).

[17] S. Bonazzola, J. Frieben, and E. Gourgoulhon, Astron. Astrophys. in press (preprint: gr-qc/9710121).

[18] J-O. Goussard, Hydrodynamique des proto-étoiles à neutrons, PhD Thesis, Université de Paris 7 (1997).

[19] J-O. Goussard, P. Haensel, and J.L. Zdunik, Astron. Astrophys. 321, 822 (1997).

[20] J-O. Goussard, P. Haensel, and J.L. Zdunik, Astron. Astrophys. 330, 1005 (1998).

[21] D. Gottlieb and S.A. Orszag, Numerical analysis of spectral methods: theory and applications (Society for Industrial and Applied Mathematics, Philadelphia, 1977).

[22] C. Canuto, M.Y. Hussaini, A. Quarteroni, T.A. Zang, Spectral methods in fluid dynamics, (Springer-Verlag, Berlin, 1988).

[23] Y. Eriguchi and E. Müller, Astron. Astrophys. 146, 260 (1985).

[24] Y. Eriguchi and E. Müller, Astron. Astrophys. 248, 435 (1991).

[25] K. Uryu and Y. Eriguchi, Mon. Not. R. Astron. Soc. 282, 653 (1996).

[26] S. Bonazzola, E. Gourgoulhon, and J-A. Marck, J. Comp. Appl. Math., in press (1998).

[27] S. Bonazzola and J-A. Marck, J. Comp. Phys. 87, 201 (1990).

[28] S. Bonazzola and E. Gourgoulhon, in Relativistic Gravitation and Gravitational Radiation, edited by J-A. Marck and J.-P. Lasota (Cambridge University Press, Cambridge, England, 1997).

[29] U.M. Schaudt and H. Pfister, Phys. Rev. Lett. 77, 3284 (1996).

[30] S. Chandrasekhar, Ellipsoidal figures of equilibrium (Yale University Press, New Haven, 1969).

[31] S. Bonazzola and E. Gourgoulhon, Class. Quantum Grav. 11, 1775 (1994).

[32] S. Bonazzola and E. Gourgoulhon, in Relativity in General, edited by J. Diaz Alonso and M. Lorente Paramo (Editions Frontières, Gif-sur-Yvette France, 1994).

[33] S. Wolfram, Mathematica, a system for doing mathematics by computer (Addison-Wesley, Reading, Massachusetts, 1992). 